\title{
Eficacia del programa de Pensamiento Prosocial en Entornos Educativos para el desarrollo de la competencia social de adolescentes en situación de riesgo de inadaptación social
}

\section{Effectiveness of Prosocial Thinking Program in Educational Environments to develop the social competence of adolescents at risk of social maladjustment}

Fecha de recepción: 23/11/2014

Fecha de aceptación: 24/11/2015
Fernández Durán, $M^{a}$ Angeles * Viguer Seguí, Paz **

Cantero López, María José **

$\left({ }^{*}\right)$ Departamento de Orientación de Escuelas de Artesanos de Valencia.

$\left(^{* *}\right)$ Departamento de Psicología Evolutiva y de la Educación. Universitat de València.

\section{resumen/ahstract:}

Resumen: La inadaptación social en la adolescencia es un factor de riesgo para el ajuste personal y social en la edad adulta. Determinadas habilidades sociocognitivas incluidas en la competencia social previenen el riesgo de desadaptación social y favorecen el desarrollo óptimo del adolescente. La escuela se presenta como un contexto ideal para desarrollar habilidades cognitivas, sociales y emocionales que favorezcan la competencia social. El objetivo de este estudio fue comprobar la eficacia del Programa de Pensamiento Prosocial en Entornos Educativos (PPSEE) para desarrollar la competencia social de adolescentes en el contexto escolar, específicamente: las habilidades de comunicación, el autocontrol emocional, la resolución de problemas, las habilidades sociales y los valores. Los participantes fueron 30 alumnos entre 12 y 15 años escolarizados de $1^{\circ}$ a $2^{\circ}$ de educación secundaria. Los resultados mostraron que los alumnos que participaron en el programa mejoraron su autocontrol, su capacidad para la resolución de problemas interpersonales y sus valores prosociales alcanzando puntuaciones similares a las del grupo control después de la intervención. Se concluye que el PPSEE resulta un programa útil para favorecer la competencia social de los alumnos de educación secundaria en situación de riesgo de inadaptación.

Abstract: Social maladjustment in adolescence is a risk factor for personal and social adjustment in adulthood. Certain socio-cognitive skills included in social competence prevent the risk of social maladjustment and promote the optimal development of the adolescent. The school is the ideal place to develop cognitive, social and emotional skills that promote social competence. The aim of this study was to test the effectiveness of Prosocial Thinking Program in Educational Environments (PPSEE) to develop the social competence of teenagers in the school context, namely: communication skills, emotional self-control, problem solving skills, social skills and values. Participants were 30 students between 12 and 15 years enrolled from 1st to 2rd grade of secondary education. The results showed that students who participated in the program improved their self-control, their ability to solve interpersonal problems and their prosocial values reaching levels similar to those of the control group after the intervention. We conclude that PPSEE is a useful program to promote social competence of high school students at risk of maladjustment.

\section{palabras clave/keywords:}

inadaptación social, competencia social, adolescencia, educación secundaria.

social maladjustment, social competence, adolescence, secondary education. 


\section{Introducción}

La competencia social ayuda al adolescente a desarrollarse en las áreas que aseguran un correcto ajuste sociopersonal, de forma que la adaptación surge como resultado de poseer y poner en práctica una serie de estrategias y habilidades consideradas culturalmente como positivas, mientras que lo contrario de esto aumenta el riesgo de conducta antisocial (López, Garrido y Ross, 2001; Carrasco y Trianes, 2010). En este sentido, diferentes enfoques teóricos y modelos de intervención han propuesto una psicología optimizadora en la que se utilizan los principios teóricos y la tecnología de intervención para la promoción del desarrollo (Viguer, 2004). Además, diversos autores, entre los que destaca Wagner (1996), estudian los distintos criterios del desarrollo óptimo adolescente y consideran el rol que los psicólogos pueden tener para potenciarlo. Este autor señala la importancia de desarrollar intervenciones dirigidas a las áreas social, emocional y cognitiva del adolescente, como estrategia básica para facilitar su desarrollo óptimo.

En el contexto español, de forma más reciente, Oliva, Ríos, Antolín, Parra, Hernando y Pertegal (2010) proponen un modelo de desarrollo positivo adolescente que incluye cinco áreas: social, cognitiva, personal, emocional y moral. Este modelo tiene una visión optimista del adolescente que considera que cuando existen condiciones favorables y los adolescentes se ven implicados en relaciones saludables con su entorno, éstos crecen como ciudadanos responsables y prosociales. Así, cuando se favorece el contexto para que el adolescente se desarrolle, no solo se contribuye a su crecimiento sino también a la mejora de la sociedad.

Desde la psicología evolutiva, y específicamente en el ámbito de la optimización evolutiva, a partir de los años noventa se ha producido un importante auge de los programas de intervención dirigidos a las áreas personal y social en las distintas etapas del desarrollo, y especialmente en la adolescencia. Entre ellos, el Programa de Pensamiento Prosocial Junior (PPSjr.) (Cano, López, Garrido y López, 1998) tenía como objetivo incrementar la competencia y la autoestima de adolescentes en situación de riesgo, previniendo la inadaptación. El programa PPSjr. consistía en un modelo de entrenamiento cognitivo-conductual cuyo objetivo era enseñar las habilidades cognitivas y actitudes para poseer una adecuada competencia social. Partía de la premisa de que estas habilidades no se dan en los adolescentes de manera espontánea, sino que deben enseñarse, siendo la escuela uno de los principales contextos para su enseñanza (López, Garrido y Ross, 2001).

La educación en las escuelas, por tanto, además de favorecer el desarrollo cognitivo debe completarse con propuestas que promuevan un adecuado desarrollo socio-emocional y, en esta línea, en las dos últimas décadas han proliferado numerosos programas de promoción de las habilidades emocionales y sociales (Caballo, 2002; Carrasco y Luna, 2001; Garaigordobil, 2000; López, Garrido, Rodríguez y Paíno, 2002; Luca de Tena, Rodríguez y Sureda, 2001; Monjas, 2002; Moraleda, 1998; Trianes, 1996; Vallés y Vallés, 1996). No cabe duda, que en la escuela se establecen relaciones interpersonales que afectan al proceso de aprendizaje, además estas relaciones pueden ser una fuente de conflictos que también se producen dentro de la sociedad, por lo que es muy importante que el alumno aprenda a manejar sus emociones y desarrolle adecuadamente sus habilidades sociales. A su vez, la adaptación so- 
cial tiene efectos positivos sobre el clima del aula, considerándose una variable muy importante para la mejora de la convivencia escolar y por supuesto, para el desarrollo integral del alumno (Carrasco y Trianes, 2010). Pese a constituir una temática con amplia tradición en el ámbito de la psicología de la educación, los programas dirigidos a prevenir problemas de inadaptación social y de mejora de las competencias personales en el ámbito escolar reciben mucha atención en la investigación actual, así como en el diseño y validación de propuestas de intervención (Caballero, Delgado y Escartí, 2013; Garaigordobil y Peña, 2014; Lab, 2015).

En este contexto, y con el objetivo de promover un desarrollo integral y óptimo de los alumnos, se planteó desde el Departamento de Orientación de un centro de Educación Secundaria, la posibilidad de iniciar una enseñanza estructurada de habilidades cognitivas y sociales que favorecieran el desarrollo de adolescentes con un mayor riesgo de inadaptación personal y social. Se eligió el Programa del Pensamiento Prosocial en Entornos Educativos (PPSEE) (Fernández-Durán, García, Garrido y López, 2005; Fernández-Durán, 2015), para cumplir ese objetivo educativo dado que se centra en el desarrollo de habilidades que favorecen la competencia social, sobre todo en alumnos en riesgo de inadaptación personal y social. Este programa deriva del programa PPSjr y considera que si bien las intervenciones educativas de tutores, profesores y padres es básica para el aprendizaje de estrategias y habilidades cognitivas que favorezcan esta competencia, para la mayoría del alumnado, y sobre todo para aquel que está en riesgo de inadaptación, es conveniente llevar a cabo una enseñanza explícita y directa de las mismas.

Los estudiantes necesitan aprender no solo el pensamiento inferencial y crítico que les permita ser competentes en determinadas áreas curriculares, sino también las habilidades cognitivas, emocionales y conductuales necesarias para su adecuada integración social. Desde este punto de vista, el programa PPSEE promueve la adquisición de estas habilidades y puede incluirse en el marco de la Ley Orgánica de Mejora de la Calidad Educativa (2013), donde se reconoce la importancia del fomento de las relaciones sociales constructivas como factor de protección y prevención.

El programa PPSEE tiene como objetivo principal trabajar la competencia social desde la educación secundaria. El programa se compone de siete módulos repartidos en 32 sesiones y utiliza una metodología activa y constructiva que permite poner en práctica las habilidades relacionadas con la competencia social. Aunque se parte de unas instrucciones previas, el alumno interactúa con el entorno participando en las tareas diseñadas, extrayendo sus propias conclusiones de aprendizaje a partir de las interacciones con los demás y de su propia experiencia, y poniendo en marcha procesos cognitivos y emocionales. Mediante dinámicas y simulaciones, el alumno extrae sus propios conocimientos de una forma reflexiva, participativa, práctica y motivadora. Se utilizan las siguientes técnicas: instrucción verbal, refuerzo, debate, relajación, autoinstrucciones, lluvia de ideas, role-playing, dilemas morales y generalización. Además de estas técnicas el programa propone la utilización del cómic como una herramienta útil para favorecer ciertas capacidades personales, como instrumento de síntesis, como elemento que muestra de forma distinta los contenidos y como elemento motivador para promover la participación del alumnado. 
Así pues, el objetivo de este estudio es comprobar la eficacia del programa Pensamiento Prosocial en Entornos Educativos para desarrollar la competencia social de adolescentes en situación de riesgo de inadaptación. Concretamente se pretende comprobar la influencia del programa en las siguientes áreas: autocontrol emocional, resolución de problemas, valores, habilidades de comunicación y habilidades sociales.

\section{Método}

\section{Participantes}

Los participantes fueron 30 alumnos de Enseñanza Secundaria Obligatoria de un centro concertado de la ciudad de Valencia. La edad promedio de los participantes fue de 12.7 años con una desviación típica de 0.7 , y un rango entre 12 y 15 años. Todos los participantes fueron varones. El 73.3\% $(n=22)$ cursaba primero de Enseñanza Secundaria Obligatoria y el 26.7\% ( $n=8)$ segundo. Estos alumnos se distribuyeron en dos grupos: (a) el grupo intervención formado por 15 alumnos que participaron en el programa, y (b) el grupo control formado por 15 alumnos que no participaron en él. La edad del grupo intervención osciló entre los 12 y 15 años $(M=13.1, D T=0.70)$ y en el grupo control entre 12 y 13 años $(M=$ $12.3, D T=0.47)$. En ambos grupos, el $73.3 \%(n=11)$ de los alumnos cursaban primero de Enseñanza Secundaria Obligatoria y un $26.7 \%(n=4)$ segundo.

Los criterios de selección para la inclusión de los alumnos en el programa fueron: (a) contradecir las reglas del reglamento de régimen interno del centro de manera frecuente, (b) dificultad de relación social, bien por el hecho de presentar falta de empatía y conductas antisociales o por presentar conductas que denotaban falta de asertividad. Esta información fue facilitada por la coordinadora de secundaria y consensuada por el equipo docente en base a las quejas habituales de los profesores, al número de partes disciplinarios, la frecuencia de incidentes entre iguales o entre el alumno y el profesorado.

El grupo control se seleccionó al azar de una lista de alumnos varones, eligiendo el mismo número de alumnos de cada curso que el grupo intervención. Es importante señalar que el grupo control no fue equivalente al grupo intervención, aunque se igualó en curso y sexo, los adolescentes del grupo control no presentaban problemas de conducta y de relación social. Esto fue así porque el programa formaba parte de un proyecto de Compensación Educativa que no permitía excluir a alumnos con dificultades de adaptación escolar. No fue posible, por tanto, disponer de un grupo control equivalente.

\section{Instrumentos}

Cuestionario de Autocontrol Infantil y Adolescente (CACIA) de Capafons y Silva (1986). Este cuestionario tiene como objetivo medir la autopercepción del autocontrol desde un punto de vista conductual. Consta de 89 ítems con una escala de respuesta de "si" o "no". agrupados en cinco escalas. En esta investigación se utilizaron dos de ellas: retroalimentación personal (RP) y retraso de la recompensa (RR). La escala de retroalimentación personal consta de 21 ítems que miden auto-observación, análisis de situaciones y búsqueda de causas del comportamiento. Si la puntuación es elevada significa que la persona se conoce a sí misma y es capaz de percibir las consecuencias de sus acciones y quiere conocer los 
motivos que llevan a actuar de una determinada forma. La escala de retraso de la recompensa (RR) consta de 19 ítems que miden la capacidad de la persona para priorizar las tareas que debe realizar dejando en último lugar aquello que le apetece. Puntuaciones elevadas indican que la persona es capaz de organizar sus tareas y no se deja llevar solo por lo que le apetece. Los índices de consistencia interna son de .79 y .71 respectivamente (Capafons y Silva, 1986).

Cuestionario de Competencia Social Escolar (COSOE, Fernández-Durán, 2015). Este cuestionario consta de 49 ítems en el que el alumno/a se autoevalúa mediante una escala tipo Likert donde "1" es nunca lo hago y "4" es siempre lo hago. Se divide en cinco escalas. En este trabajo se utilizaron cuatro de ellas: (a) Habilidades de comunicación (HC) compuesta por 10 ítems. Una puntuación alta en esta escala significa que el alumno/a pone en práctica dentro del entorno escolar una serie de habilidades para demostrar al compañero/a mediante lenguaje verbal y no verbal que sigue su conversación y le escucha. (b) Habilidades Sociales (HS) que consta de 10 ítems. Una puntuación alta significa que el alumno/a utiliza una serie de habilidades en su relación con los demás compañeros como son: ponerse en lugar del otro, iniciar, mantener y finalizar conversaciones, expresar afecto, compartir, disculparse, negociar, realizar cumplidos, prestar ayuda y dar las gracias. (c) Resolución de Problemas (RP) formada por 13 ítems. Una puntuación alta significa que el alumno/a es capaz de poner en práctica las diferentes habilidades siguiendo las diferentes etapas dentro de la resolución de problemas interpersonales (reconocer señales internas y externas que le indican que tiene un problema, pensar en varias soluciones posibles, analizar las consecuencias de cada solución teniendo en cuenta el tiempo y la implicación para uno mismo y para los demás, elegir la mejor opción y planificar cómo llevarla a cabo. (d) Valores (VA) compuesta por 8 ítems. Una puntuación alta significa que el alumno/a pone en práctica conductas que tienen que ver con determinados valores de reconocimiento de los demás, tolerancia, compromiso, respeto y cooperación. El COSOE muestra adecuados índices de consistencia interna, con valores de $\alpha$ de Cronbach de .78 para la escala de habilidades de comunicación, de .83 para la escala de habilidades sociales, de .90 para la escala de resolución de problemas y de .78 para la escala de valores (Fernández-Durán, 2015).

\section{Diseño}

Se ha utilizado un diseño cuasi-experimental, en concreto un diseño de grupo de control no equivalente. Este diseño incluye un grupo intervención (al que se aplica el programa) y otro control (al que no se le aplica el programa). Ambos grupos han sido evaluados en dos momentos temporales, pretest y postest, pero sin poseer equivalencia preexperimental de muestreo.

Con la intención de analizar la eficacia del PPSEE para desarrollar diferentes áreas de la competencia social de los alumnos se llevaron a cabo análisis univariados de varianza (ANOVA) de medidas repetidas (MR) 2x2, el grupo (intervención-control) fue la variable entresujeto y el tiempo (pretest-postest) la variable intrasujetos. En los casos de incumplimiento del supuesto de normalidad se utilizaron las pruebas no paramétricas $U$ de MannWhitney para grupos independientes y $W$ de Wilcoxon para muestras relacionadas. 


\section{Resultados}

La variable Autocontrol evaluada con el Cuestionario de Autocontrol Infantil y Adolescente (CACIA) mediante la escala de Retroalimentación Personal (RP) mostró un efecto significativo de la variable tiempo $\left(F(1,27)=7.192, p=.01, \eta_{p}^{2}=.21\right)$ indicando que el autocontrol de los alumnos varió significativamente a través del tiempo, aumentando el nivel de esta variable de la fase pretest a la fase postest (media estimada pretest $=0.62$; media estimada postest $=0.69$ ) con un tamaño del efecto grande. También se encontró un efecto significativo en la variable interacción tiempo x grupo con un tamaño del efecto grande $(\lambda$ $\left.=.80, F(1,27)=6.478, p=.01, \eta_{p}^{2}=.19\right)$. El análisis de los efectos simples de la interacción mostró diferencias significativas para el grupo intervención entre el momento pretest y postest (dif. Medias $=-.13, F(1,27)=14.148, p<.01, \eta_{p}^{2}=.34, d=0.85$ ), es decir, el grupo intervención mejoró significativamente en autocontrol tras la intervención con el programa. El tamaño del efecto de las diferencias fue alto $(d$ de Cohen $=0.85)$. Esta diferencia queda reflejada en la Figura 1.

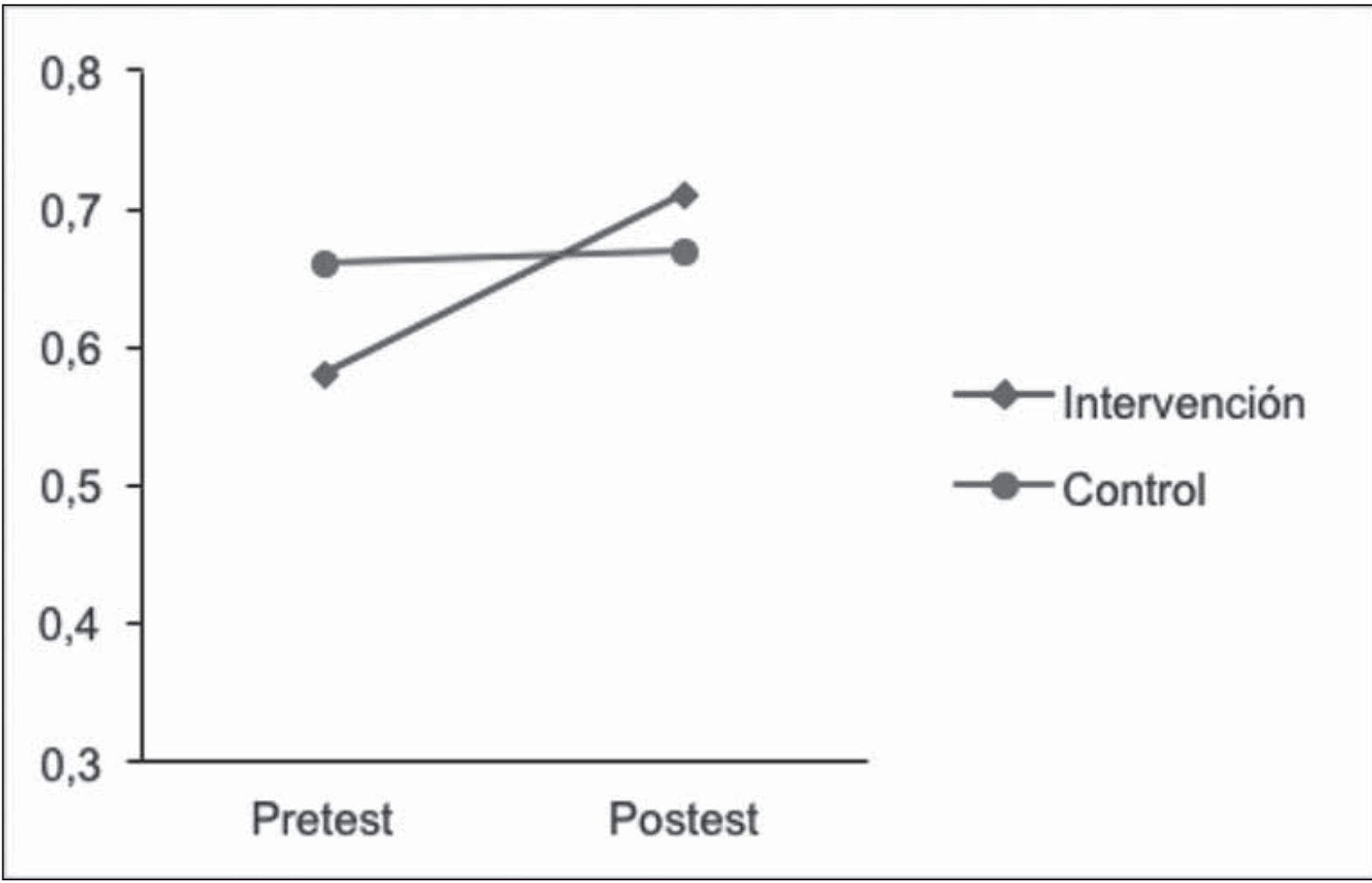

Figura 1. Evolución en autocontrol, retroalimentación personal, en los grupos intervención y control.

Por otro lado, la variable Autocontrol evaluada con el Cuestionario de Autocontrol Infantil y Adolescente (CACIA) mediante la escala Retraso de la Recompensa (RR) mostró un efecto significativo de la variable tiempo $\left(F(1,27)=14.691, p<.01, \eta_{p}^{2}=.32\right)$ lo que indica que el autocontrol de los alumnos, concretamente su capacidad para retrasar la recompensa, varió significativamente a través del tiempo, aumentando el nivel de esta variable de la fase pretest a la fase postest (media estimada pretest $=0.51$; media estimada postest $=0.58$ ) con un tamaño del efecto grande. También encontramos un efecto significativo de la variable 
grupo $\left(F(1,27)=8.037, p<.01, \eta_{\mathrm{p}}^{2}=.22\right)$ indicando diferencias entre los grupos intervención y control en autocontrol (media estimada grupo intervención $=0.45$; media estimada grupo control $=0.64$ ), siendo mayor en el grupo control, con un gran tamaño del efecto. Por último, se encontró un efecto significativo en la variable interacción tiempo x grupo con un tamaño del efecto grande $\left(\lambda=.69, F(1,27)=11.855, p<.01, \eta^{2}=.19\right)$. Respecto a los efectos simples de la interacción, el estudio de las medidas pre mostró diferencias significativas entre las medias del grupo intervención y el control (dif. Medias $=-0.26, F(1,27)=15.180$, $p<.01, d=2.87)$, lo que indicó que el grupo control mostró mayor autocontrol, concretamente en su capacidad de retraso de la recompensa, que el grupo intervención, antes de la aplicación del programa. Esta diferencia obtuvo un gran tamaño del efecto. Por otro lado, el grupo intervención mostró diferencias significativas entre el momento pretest y postest (dif. Medias $=-0.14, F(1,27)=27.416, p<.01, d=0.26$ ) con un tamaño del efecto bajo. El grupo intervención mejoró significativamente en autocontrol concretado en su capacidad para retrasar la recompensa como consecuencia de la intervención con el programa. Las diferencias encontradas quedan reflejadas en la Figura 2.

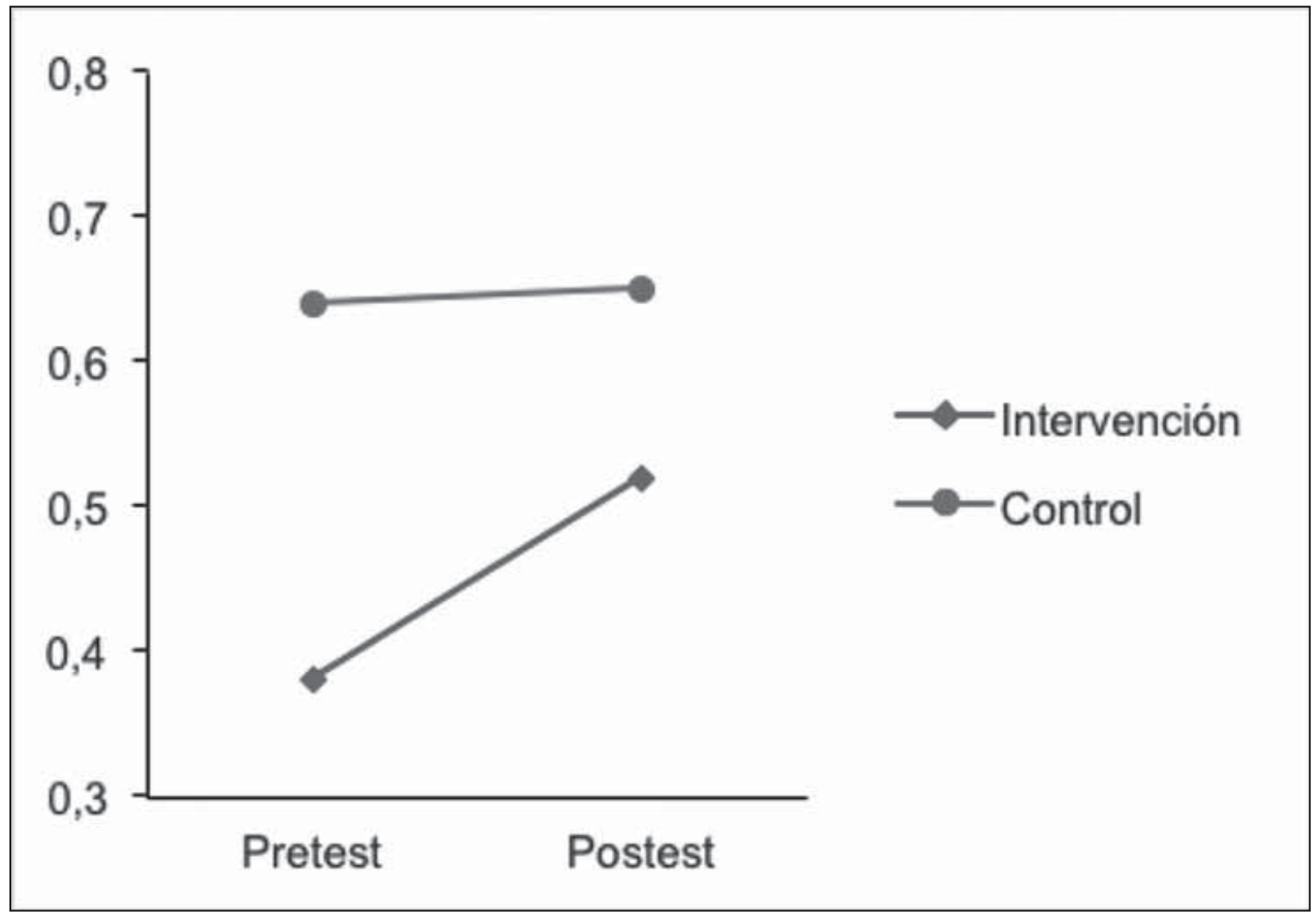

Figura 2. Evolución en autocontrol, retraso de la recompensa, en los grupos intervención y control.

La variable Resolución de problemas evaluada con el COSOE mostró un efecto significativo de la variable tiempo $\left(F(1,27)=12.667, p<.01, \eta_{\mathrm{p}}^{2}=.32\right)$ lo que nos indica que la percepción de los alumnos sobre su capacidad de resolución de problemas en el contexto escolar varió significativamente a través del tiempo, aumentando el nivel de esta variable de la fase pretest a la fase postest $($ media estimada pretest $=2.54$; media estimada postest $=$ 
2.89) con un tamaño del efecto grande. A la vez, encontramos un efecto significativo de la variable grupo $\left(F(1,27)=4.623, p=.04, \eta_{\mathrm{p}}^{2}=.14\right)$ indicando diferencias entre los grupos intervención y control en la percepción de resolución de problemas (media estimada grupo intervención $=2.55$; media estimada grupo control $=2.88$ ), siendo mayor en el grupo control, con un efecto del tamaño grande. Por último, se encontró un efecto significativo en la variable interacción tiempo $\mathrm{x}$ grupo con un tamaño del efecto grande $(\lambda=.73, F(1,27)=$ 9.746, $p<.01, \eta_{\mathrm{p}}^{2}=.26$ ). Los resultados de los efectos simples de la interacción tiempo $\mathrm{x}$ grupo en el estudio de las medidas pre mostró diferencias significativas entre medias del grupo intervención y el control (dif. Medias $=-0.64, F(1,27)=11.277, p<.01, d=4.73$ ), esto indica que el grupo control mostró una mejor percepción sobre su capacidad de resolución de problemas en el contexto escolar, que el grupo intervención, antes de la aplicación del programa además esta diferencia mostró un gran tamaño del efecto. En la interacción grupo x tiempo encontramos que el grupo intervención mostró diferencias significativas entre el momento pretest y postest (dif. Medias $=-0.67, F(1,27)=23.115, p<.01, d=5.27$.), por lo que podemos afirmar que el grupo intervención mejoró significativamente en la percepción de su capacidad de resolución de problemas como consecuencia de la intervención con el programa. Las diferencias encontradas quedan reflejadas en la Figura 3.

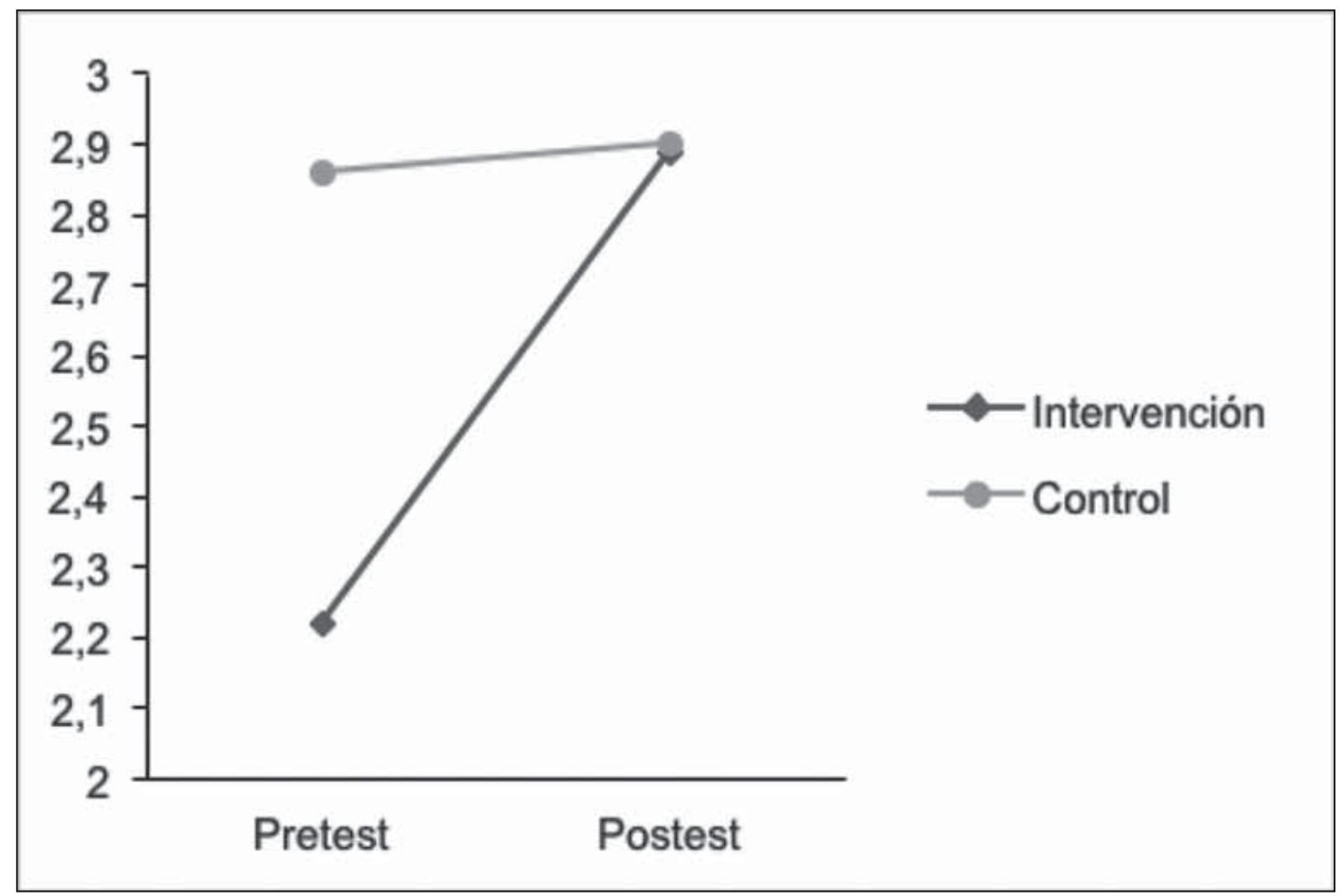

Figura 3. Evolución en Resolución de Problemas en los grupos intervención y control.

Para la variable Valores evaluada con el COSOE, la prueba U de Mann Whitney indicó diferencias significativas entre el grupo intervención y el grupo control en la fase pretest ( $M$ intervención $=3.07, D T=.55, M$ control $=3.50, D T=.36, U=.55, p=.04)$. El grupo control puntuó significativamente más alto en la práctica de conductas que tienen que ver 
con determinados valores de reconocimiento de los demás, tolerancia, compromiso, respeto y cooperación en el momento previo a la intervención. Por otro lado, también encontramos diferencias significativas entre el grupo intervención y el grupo control en la fase postest ( $M$ intervención $=3.40, D T=.56, M$ control $=3.48, D T=.38, U=58.500, p=.04$ ), en el mismo sentido. Por otra parte, la prueba de rangos con signos de Wilcoxon mostró que existía diferencia significativa entre el momento pretest y postest para el grupo intervención, siendo la media mayor en el momento postest $(M$ pretest $=3.07 ; D T=.55 ; M$ postest $=3.40$, $D T=.56, z=-2.42, p=.01$ ), es decir, el grupo intervención mejoró en la percepción sobre sus valores después de participar en el programa. Por otro lado, no se obtuvieron diferencias entre el momento pre y post en el grupo control. Las diferencias analizadas quedan reflejadas en la Figura 4.

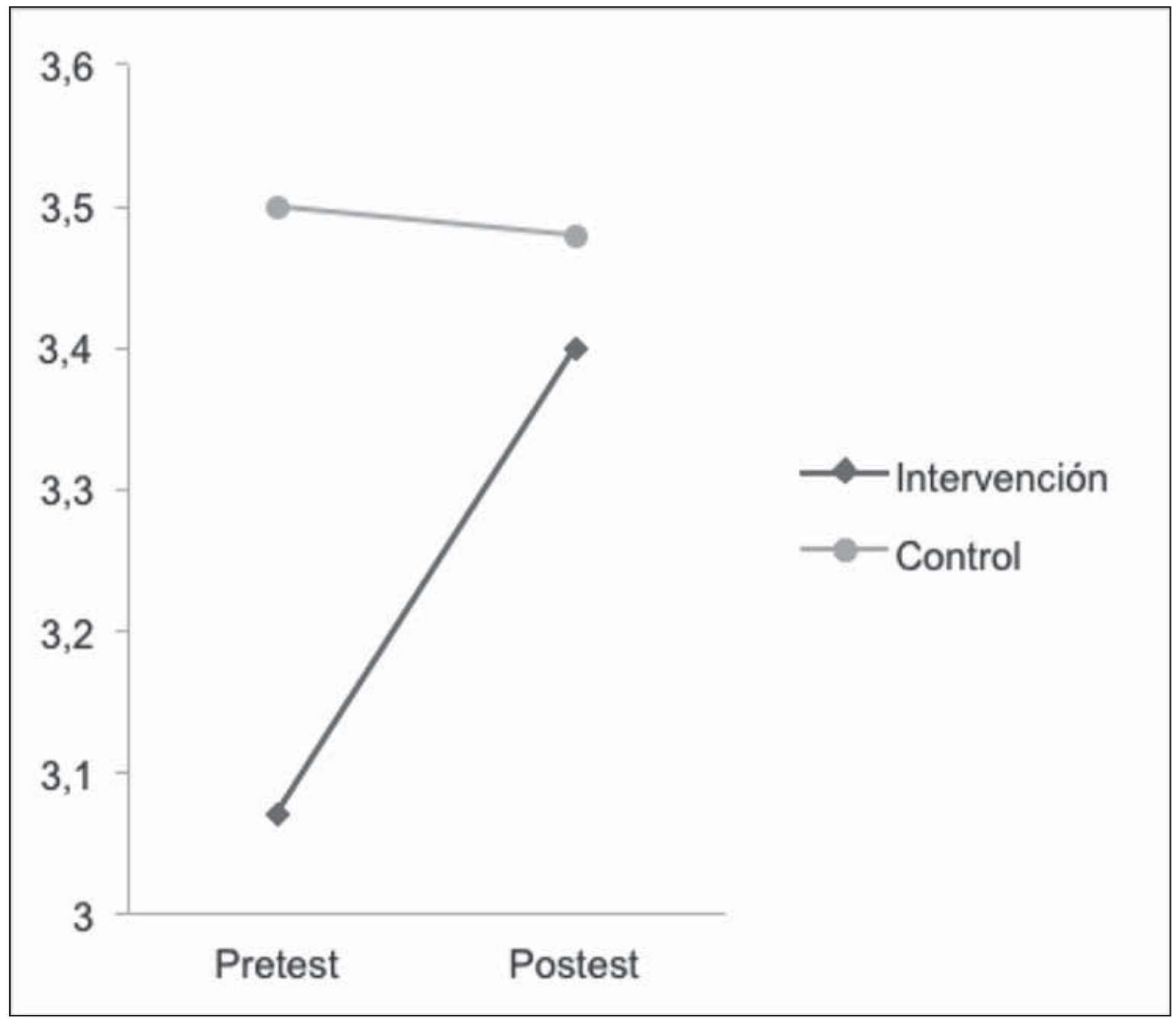

Figura 4. Evolución en Valores en los grupos intervención y control.

Con respecto a las variable Habilidades de Comunicación no se obtuvieron diferencias significativas ni en la variable tiempo $\left(\lambda=.81, F(1,27)=6.008, p=.21, \eta_{\mathrm{p}}^{2}=.18\right)$, ni en la variable grupo $\left(F(1,27)=2.004, p=.16, \eta_{\mathrm{p}}^{2}=.69\right)$, ni en la interacción entre ambas $(\lambda=.95, F$ $\left.(1,27)=1.174, p=.28, \eta_{\mathrm{p}}^{2}=.42\right)$. El mismo resultado se observó en la variable Habilidades Sociales, que no mostró resultados significativos ni en la variable tiempo $(\lambda=.94, F(1,27)$ 
$\left.=1.692, p=.20, \eta_{\mathrm{p}}^{2}=.59\right)$, ni en la variable grupo $\left(F(1,27)=1.576, p=.22, \eta_{\mathrm{p}}^{2}=.05\right)$, ni en la interacción $\left(\lambda=.99, F(1,27)=.058, p=.81, \eta_{\mathrm{p}}^{2}=.00\right)$.

\section{Discusión}

El objetivo de esta investigación es comprobar la eficacia del programa Pensamiento Prosocial en Entornos Educativos para desarrollar la competencia social de un grupo de adolescentes de Educación Secundaria en situación de riesgo de inadaptación social. En concreto, se pretende comprobar el cambio en autocontrol emocional (retroalimentación personal y retraso de la recompensa), resolución de problemas, valores, habilidades de comunicación y habilidades sociales comparando los resultados con un grupo control antes y después de la implementación del PPSEE.

En primer lugar, la variable Autocontrol evaluada a través de la retroalimentación personal mostró cambios en los alumnos que participaron en el programa. El grupo intervención aumentó su capacidad de conocerse a sí mismos y su capacidad de percibir las consecuencias de sus acciones, queriendo conocer los motivos que les llevan a actuar de una determinada manera. Del mismo modo, respecto a la variable Autocontrol evaluada a través del retraso de la recompensa se obtuvieron cambios positivos en los alumnos que participaron en el programa. Los estudiantes con problemas de inadaptación mejoraron su capacidad para retrasar la recompensa, siendo más capaces de organizar sus acciones sin dejarse llevar solo por el presente inmediato y la comodidad. Es interesante señalar que al comparar las puntuaciones entre ambos grupos antes de la aplicación del programa, el grupo tratamiento mostraba mayores dificultades en el retraso de la recompensa que el grupo control, igualándose las puntuaciones entre ambos grupos como consecuencia de la intervención en el grupo de adolescentes en situación de riesgo. Estudios similares realizados en otros contextos, han encontrado también que la intervención educativa a través de programas sistemáticos ha promovido una mejora de la retroalimentación personal y del retraso de la recompensa en adolescentes (Cecchini, Fernández, González y Arruza, 2008; Cecchini, Montero y Peña, 2003).

En segundo lugar, respecto a la variable Resolución de Problemas los alumnos que intervinieron en el programa mejoraron significativamente su capacidad para reconocer señales internas y externas que indican que se tiene un problema, en su capacidad para pensar en varias soluciones posibles, analizando las consecuencias de cada solución y en su capacidad para elegir la mejor opción, planificándola para llevarla a cabo. En relación a la comparación entre el grupo intervención y el control, se observaron diferencias en la evaluación inicial en el sentido esperado, siendo la capacidad de resolución de problemas menor en el grupo intervención. Como consecuencia de la aplicación del programa, el grupo de adolescentes en situación de riesgo de inadaptación social mostró puntuaciones similares a las del grupo control en el segundo momento de medida. El aumento en la capacidad de resolución de problemas como consecuencia de la aplicación de un programa de competencia social es un efecto que también fue observado en anteriores investigaciones (Fernández-Durán, 2004; López, Garrido y Ross, 2001). 
En tercer lugar, se observaron los efectos esperados en los alumnos que participaron en el programa respecto a su percepción en valores. En el momento previo a la aplicación del programa, el grupo intervención obtuvo una menor puntuación en valores que el grupo control. Sin embargo, tras la aplicación del programa los adolescentes mejoraron su percepción respecto a los valores relativos al respeto hacia los demás, la tolerancia, el compromiso y la cooperación igualándose sus puntuaciones con el grupo control. En los últimos años, encontramos programas de intervención educativa dedicados a la promoción de valores que están logrando buenos resultados trabajando específicamente a través de la actividad física y el deporte (Jiménez y Durán, 2004).

Por último, en las habilidades de comunicación y en las habilidades sociales no se obtuvieron cambios significativos en el sentido esperado en los alumnos que participaron en el programa no igualándose sus puntuaciones con las del grupo control. Consideramos que este resultado puede ser debido al pequeño tamaño muestral, una de las principales limitaciones del estudio. Somos conscientes de que se requieren nuevos trabajos en los que se implemente el programa a un número mayor de participantes. Además, en la medida de lo posible sería aconsejable mejorar el diseño aplicando el programa a dos grupos de adolescentes en situación de riesgo de inadaptación, puesto que en este estudio al utilizar un diseño cuasiexperimental de grupo no equivalente, lo que esperábamos era que los dos grupos (intervención y control) se igualarán al finalizar la aplicación del programa.

Así pues, podemos concluir que el programa PPSEE, a pesar de las limitaciones comentadas, ayuda a mejorar la retroalimentación personal y la capacidad de retrasar la recompensa, ayuda también en la resolución de problemas y permite afianzar valores prosociales en el contexto escolar. Por tanto, el PPSEE se presenta como una nueva propuesta de intervención para favorecer el desarrollo positivo del adolescente, que se aplica de una forma estructurada durante todo el curso escolar y queda abierto a futuras modificaciones con el fin de desarrollar la competencia social de los adolescentes en situación de riesgo de inadaptación social y personal.

\section{Referencias}

Caballero, P., Delgado, M. A. y Escartí, A. (2013). Analysis of Teaching Personal and Social Responsibility model-based programmes applied in USA and Spain. Journal of Human Sport and Exercise 8(2), 427-441.

Caballo, V. E. (2002). Manual de evaluación y entrenamiento de las habilidades sociales. Madrid: Siglo XXI de España.

Capafons, A. y Silva, A. (1986). Cuestionario de autocontrol infantil y adolescente CACIA. Madrid: TEA Ediciones.

Carrasco, C. y Trianes, M. V. (2010). Clima social, prosocialidad y violencia como predictores de inadaptación escolar en primaria. European Journal of Education and Psychology, 3(2), 229-242.

Carrasco, T. J. y Luna, M. (2001). Habilidades para la vida. Madrid: Paraninfo.

Cecchini, J. A., Montero, J. y Peña, J. V. (2003). Repercusiones del programa de intervención para desarrollar la responsabilidad personal y social de Hellison sobre los comportamientos de fair-play y el auto-control. Psicothema, 15(4), 631-637.

Cecchini, J. A., Fernández, J., González, C., Arruza, J. A. (2008). Repercusiones del programa delfos de educación en valores a través del deporte en jóvenes escolares. Revista de Educación, 346, 167-186. 
Fernández-Durán, M. A (2015). Desarrollo de la competencia social en adolescentes: creación, aplicación y análisis del programa El Pensamiento Prosocial en Entornos Educativos (Tesis doctoral). Universitat de València, Valencia.

Fernández-Durán, M. A, García, F., Garrido, V., y López, M. J. (2005). El Programa de Pensamiento Prosocial en Entornos Educativos. En V. Garrido y M. J. López, Manual de intervención educativa en readaptación social. Vol2. Los programa del pensamiento prosocial (pp. 311-492).Valencia: Tirant lo Blanch.

Garaigordobil, M. (2000). Intervención psicológica con adolescentes. Un programa para el desarrollo de la personalidad y la educación en derechos humanos. Madrid: Pirámide.

Garaigordobil, M. y Peña, A. (2014). Intervención en las habilidades sociales: efectos en la inteligencia emocional y la conducta social. Psico logía conductual: Revista internacional de psicología clínica y de la salud, 22(3), 551-567.

Jiménez, P. J. y Durán, L. J. (2004). Propuesta de un programa para educar en valores a través de la actividad física y el deporte. Ciencias aplicadas a la actividad física y el deporte, 77, 25-29.

López, M. J., Garrido, V. y Ross, R. (2001). El programa del pensamiento prosocial: avances recientes. Valencia: Tirant lo Blanch.

López, M. J., Garrido, V., Rodríguez, F. J. y Paino, S. G. (2002). Jóvenes y competencia social: un programa de intervención. Psicothema, 14 Supl., 155-163.

Luca de Tena, C., Rodríguez, R. I. y Sureda I. (2001). Programa de habilidades sociales en la E.S.O. Málaga: Aljibe.

Monjas, M. I. (2002). Programa de enseñanza de habilidades de interacción social (PEHIS). Madrid: CEPE.

Moraleda, M. (1998). Educar en la competencia social. Un programa para la tutoría con adolescentes. Madrid: C.C.S.

Oliva, A., Ríos. M., Antolín. L., Parra. A., Hernando. A. y Pertegal. M. A. (2010). Más allá del déficit: construyendo un modelo de desarrollo positivo adolescente. Infancia y Aprendizaje, 33(2), 223-234.

Trianes, M. V. (1996). Educación y competencia social. Un programa en el aula. Málaga: Aljibe.

Vallés, A. y Vallés, C. (1996). Las habilidades sociales en la escuela. Una propuesta curricular. Madrid: EOS.

Viguer, P. (2004). Optimización evolutiva. Fundamentos del desarrollo óptimo. Madrid: Pirámide.

Wagner, W. G. (1996). Optimal development in adolescence: What is it and how can it be encouraged. The Counseling Psychologist, 24(3), 360-399. 